\title{
Impact of early fundoplication or gastrostomy tube on midterm outcomes for patients with single ventricle
}

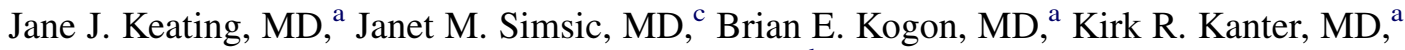 \\ Jeryl Huckaby, RRT, CCRC, ${ }^{\mathrm{c}}$ Patrick D. Kilgo, MS, ${ }^{\mathrm{b}}$ and Paul M. Kirshbom, MD ${ }^{\mathrm{a}}$
}

\begin{abstract}
Objective: Patients with single-ventricle heart disease experience early and late failure during and after staged palliation. Little is known about the factors related to continued risk of transplantation and mortality after completion of staged palliation. The long-term outcome of patients with single-ventricle disease who require a gastric fundoplication early in life has not been assessed.
\end{abstract}

Methods: A total of 155 patients with single-ventricle disease who survived their first-stage palliative procedure were enrolled in a research registry. Demographic and anatomic variables were collected, and the families were contacted every 6 months for prospective documentation of transplant-free survival. Medical records were reviewed for the details of noncardiac surgical procedures. Univariate and multivariate regression analyses were performed to determine the impact of early gastric surgery on late transplant-free survival.

Results: There were 93 male patients, median gestational age was 38 weeks, and birth weight was $3.2 \mathrm{~kg}$. Sixtyfive patients $(42 \%)$ had hypoplastic left heart syndrome. Twelve patients $(7.7 \%)$ had a genetic syndrome. Thirty-two patients $(21 \%)$ had a fundoplication or a gastrostomy tube at less than 2 years of age. Median follow-up was 4.3 years (range, 79 days to 10 years). Race, gender, gestational age, birth weight, and genetic syndrome did not alter midterm transplant-free survival. Need for fundoplication or gastrostomy was an independent risk factor for decreased transplant-free survival $(P=.003$; hazard ratio, 4.29), which was unchanged when adjusted for all covariates.

Conclusions: The need for early fundoplication or gastrostomy is associated with decreased transplant-free survival for patients with palliated single-ventricle heart disease. (J Thorac Cardiovasc Surg 2012;143:891-5)

Earn CME credits at

http://cme.ctsnetjournals.org

Widespread clinical evidence has demonstrated improving outcomes for infants born with single-ventricle disease, including hypoplastic left heart syndrome (HLHS). ${ }^{1,2}$ Specifically, several studies have shown reduced early mortality after both the Norwood and Fontan procedures over the past 2 decades. ${ }^{2-4}$ Despite improved early survival, patients with single-ventricle heart disease have been shown to have ongoing attrition long after Fontan completion. ${ }^{3}$ To date, little is known about the factors related to the continued

\footnotetext{
From the Division of Cardiothoracic Surgery ${ }^{\mathrm{a}}$ and Department of Biostatistics, ${ }^{\mathrm{b}}$ Rollins School of Public Health, Emory University School of Medicine, Atlanta, $\mathrm{Ga}$; and Children's Healthcare of Atlanta, ${ }^{\mathrm{c}}$ Atlanta, Ga.

Disclosures: Authors have nothing to disclose with regard to commercial support.

Read at the 37th Annual Meeting of The Western Thoracic Surgical Association, Colorado Springs, Colorado, June 22-25, 2011.

Received for publication June 21, 2011; revisions received Nov 11, 2011; accepted for publication Dec 14, 2011; available ahead of print Jan 30, 2012.

Address for reprints: Paul M. Kirshbom, MD, 1405 Clifton Road, Atlanta, GA 30322 (E-mail: pkirshb@emory.edu)

$0022-5223 / \$ 36.00$

Copyright (C) 2012 by The American Association for Thoracic Surgery doi:10.1016/j.jtcvs.2011.12.019
}

risk of transplantation and mortality occurring after the completion of staged palliation.

Children with single-ventricle disease are complex patients even beyond the scope of their cardiovascular pathology. As a result, they often require noncardiac surgery. The most commonly performed noncardiac surgical procedures in this population are Nissen fundoplication, gastrostomy tube (GT) placement, circumcision, and partial resection of the small intestine. ${ }^{5}$ The literature is contradictory with regard to the perioperative risk of noncardiac procedures in this patient population, with reports ranging between zero mortality for laparoscopic procedures and $19 \%$ mortality associated with even minor procedures. ${ }^{5,6}$ More important, the long-term outcome of patients with singleventricle disease who require noncardiac surgery early in life has not been reported.

The purpose of this study was to investigate the effect of early Nissen fundoplication or GT on the midterm outcomes for children with single-ventricle heart disease.

\section{MATERIALS AND METHODS}

Institutional review board approval was obtained to create a registry of patients with single-ventricle heart disease born between May 1999 and September 2007 who presented to Children's Healthcare of Atlanta at Egleston for cardiac catheterization before second-stage palliation. All of the patients were survivors of first-stage palliation. Patients who underwent primary transplant rather than staged palliation and those whose parents refused consent were excluded from the study. 


\section{Abbreviations and Acronyms \\ GERD $=$ gastroesophageal reflux disease \\ GT $=$ gastrostomy tube \\ HLHS $=$ hypoplastic left heart syndrome \\ $\mathrm{HR}=$ hazard ratio}

Demographic data were collected, including gender, race, cardiac diagnosis, presence of a genetic syndrome, gestational age, and birth weight. We also determined the dates of fundoplication or GT procedures. Family members were contacted every 6 months for prospective follow-up of clinical status. The typical timing of staged palliation at Children's Healthcare of Atlanta includes second-stage palliation (bidirectional Glenn shunt or hemi-Fontan procedure) at 4 to 6 months of age followed by Fontan completion (lateral tunnel or extracardiac conduit, generally fenestrated in either case) between 2 and 4 years of age. The timing and type of cardiac surgical procedures chosen were determined by surgeon and cardiologists' preferences. The indications for and timing of noncardiac procedures were abstracted from the medical record.

\section{Statistical Analysis}

An initial bivariate analysis was performed to identify (1) risk factors associated with an increased incidence of fundoplication or GT and (2) risk factors associated with long-term transplant-free survival (using Kaplan-Meier analysis and Cox proportional hazards regression models). To estimate the independent effect of fundoplication or GT on survival, we constructed a multivariable extended Cox model that adjusted for all potential confounders determined to be associated with both incidence of fundoplication or GT and long-term transplant-free survival. Fundo/GT was modeled as a time-dependent covariate, because it is a variable whose value can change over the course of the study period. Hazard ratios (HRs) and $95 \%$ confidence intervals were estimated for each effect, including the primary study effect (fundoplication or GT).

All statistical tests were evaluated at the 0.05 alpha level. Analyses were performed using SAS version 9.2 (SAS Institute Inc, Cary, NC). No adjustments for multiple comparisons were made.

\section{RESULTS}

Of the 155 patients, 32 (21\%) had a fundoplication or GT before 2 years of age. Patient demographics are shown in Table 1 . It should be noted that patients were not routinely evaluated by a geneticist. Genetic evaluation was initiated on clinical grounds, such as multiorgan anomalies or the presence of dysmorphic features. Also included in Table 1 is the bivariate analysis of association between these preoperative variables and the performance of a fundoplication or GT. Ventricular morphology and cardiac diagnosis were significantly related to the incidence of fundoplication or GT $(P=.008$ and .002 , respectively) with a greater need for fundoplication or GT in patients with a dominant right ventricle in general or HLHS in particular.

\section{Indication, Timing, and Type of Gastric Surgery}

The majority of patients who underwent gastric surgery $(24 / 32,75 \%)$ had gastroesophageal reflux disease (GERD) documented as one of the primary clinical indications.
TABLE 1. Patient demographics and preoperative clinical characteristics

\begin{tabular}{lcccc}
\hline \multicolumn{1}{c}{ Cherall } & No fundo/GT & Fundo/GT & $\boldsymbol{P}$ \\
varacteristic & $\mathbf{N}=\mathbf{1 5 5}$ & $\mathbf{N}=\mathbf{1 2 3}$ & $\mathbf{N}=\mathbf{3 2}$ & value \\
\hline Race & & & & \\
- White (\%) & $94(61)$ & $76(62)$ & $18(56)$ & .84 \\
- Black (\%) & $36(23)$ & $28(23)$ & $8(25)$ & \\
- Hispanic (\%) & $25(16)$ & $19(16)$ & $6(19)$ & \\
Female gender (\%) & $62(60)$ & $46(37)$ & $16(50)$ & .19 \\
Ventricular morphology & & & & \\
- Left (\%) & $56(36)$ & $51(42)$ & $5(16)$ & .008 \\
- Right (\%) & $88(57)$ & $62(51)$ & $26(81)$ & \\
- Both (\%) & $10(7)$ & $9(7)$ & $1(3)$ & \\
Diagnosis* & & & & \\
- HLHS (\%) & $65(42)$ & $41(33)$ & $24(75)$ & .002 \\
- DILV (\%) & $17(11)$ & $16(13)$ & $1(3)$ & \\
- Hypo RV/TA/PA (\%) & $30(20)$ & $3(22)$ & $3(9)$ & \\
- Unbalanced & $8(5)$ & $7(6)$ & $1(3)$ & \\
$\quad$ CACV (\%) & & & & \\
- Heterotaxy (\%) & $9(6)$ & $7(6)$ & $2(6)$ & \\
- Other (\%) & $25(16)$ & $24(20)$ & $1(3)$ & \\
HLHS (\%) & $65(42)$ & $41(33)$ & $24(75)$ & $<.001$ \\
Gestational age (SD)* & $38.2(2.0)$ & $38.2(2.1)$ & $38.1(1.8)$ & .71 \\
Birth weight (SD)* & $3.2(0.6)$ & $3.2(0.6)$ & $3.2(0.5)$ & .70 \\
Genetic syndrome (\%) & $12(8)$ & $9(7)$ & $3(9)$ & .70 \\
\hline
\end{tabular}

HLHS, Hypoplastic left heart syndrome; DILV, double-inlet left ventricle; hypo $R V / T A / P A$, hypoplastic right ventricle with tricuspid or pulmonary atresia; $C A V C$, complete atrioventricular canal defect; fundo/GT, fundoplication or gastrostomy tube. *Contains some missing data.

Many of these patients also had evidence of poor oral intake, failure to thrive, or aspiration documented on swallow studies. Seven patients $(22 \%)$ had a GT placed without a fundoplication for feeding intolerance or aspiration without clinical evidence of GERD, and 1 patient had a GT placed after repair of a perforated esophagus. There was no difference in midterm outcomes between the patients whose primary indication for surgery was GERD versus others $(P=.8)$.

Preoperative evaluation before gastric surgery was highly variable and determined by the cardiologist, cardiac surgeon, pediatrician, and general surgeon involved with the patient. The pediatric general surgeons generally requested an upper gastrointestinal contrast study to document the gastric anatomy and normal gastric emptying before fundoplication, which was performed in 25 of 32 patients $(78 \%)$. A pH probe study was performed in 13 of 32 patients $(40 \%)$; however, many of these studies were inconclusive because of the concurrent use of gastric acid suppressive therapy and a negative $\mathrm{pH}$ probe study did not preclude the performance of a fundoplication based on clinical evidence of GERD. Eight of the 32 patients $(25 \%)$ had documented aspiration on a swallow study before their gastric surgery. The majority of gastric procedures were performed laparascopically (29/ $32,91 \%$ ) with 1 open procedure and 2 percutaneous GTs.

The gastric surgery procedures were performed at a median age of 50 days (range, 30-726 days). Fifteen of these 
TABLE 2. Degree of association between preoperative variables and time to event survival probabilities

\begin{tabular}{|c|c|c|c|c|c|}
\hline Characteristic & 1-y survival & 5-y survival & 8-y survival & $P$ value & HR $(95 \%$ confidence interval $)$ \\
\hline \multicolumn{6}{|l|}{ Race } \\
\hline - White (ref) & 0.957 & 0.900 & 0.900 & .28 & 1 \\
\hline - Black & 0.972 & 0.912 & 0.810 & & $0.92(0.25-3.42)$ \\
\hline - Hispanic & 0.960 & 0.787 & 0.787 & & $2.25(0.75-6.72)$ \\
\hline \multicolumn{6}{|l|}{ Gender } \\
\hline - Male (ref) & 0.957 & 0.856 & 0.856 & .36 & 1 \\
\hline • Female & 0.967 & 0.934 & 0.879 & & $0.62(0.22-1.75)$ \\
\hline \multicolumn{6}{|l|}{ Ventricle morphology } \\
\hline - Left (ref) & 0.982 & 0.923 & 0.873 & .55 & 1 \\
\hline • Right & 0.955 & 0.880 & 0.880 & & $1.31(0.45-3.85)$ \\
\hline • Both & 0.900 & 0.750 & 0.750 & & $2.46(0.48-12.7)$ \\
\hline \multicolumn{6}{|l|}{ Diagnosis } \\
\hline • HLHS (ref) & 0.954 & 0.852 & 0.791 & .36 & 1 \\
\hline • DILV & 1.000 & 1.000 & 1.000 & & - \\
\hline - Hypo RV/TA/PA & 0.967 & 0.900 & 0.900 & & $0.63(0.17-2.29)$ \\
\hline - Unbalanced CAVC & 0.875 & 0.729 & 0.729 & & $1.43(0.31-6.57)$ \\
\hline - Heterotaxy & 1.00 & 0.857 & 0.857 & & $0.69(0.09-5.39)$ \\
\hline - Other & 0.960 & 0.960 & 0.960 & & $0.25(0.03-1.93)$ \\
\hline \multicolumn{6}{|l|}{ HLHS } \\
\hline - No (ref) & 0.966 & 0.912 & 0.912 & .13 & 1 \\
\hline • Yes & 0.954 & 0.852 & 0.791 & & $2.06(0.78-5.43)$ \\
\hline Gestational age (SD) & - & - & - & .94 & $0.99(0.78-1.25)$ \\
\hline Birth weight (SD) & - & - & - & .053 & $0.48(0.78-1.25)$ \\
\hline \multicolumn{6}{|l|}{ Genetic syndrome $(\%)$} \\
\hline • No (ref) & 0.965 & 0.905 & 0.874 & .54 & 1 \\
\hline • Yes & 0.917 & 0.802 & 0.802 & & $1.57(0.36-6.90)$ \\
\hline \multicolumn{6}{|l|}{ Fundo/GT } \\
\hline - No (ref) & 0.967 & 0.921 & 0.921 & & 1 \\
\hline - Yes & 0.938 & 0.756 & 0.648 & .003 & $4.29(1.65-11.14)$ \\
\hline
\end{tabular}

Shown is transplant-free survival at several time points associated with each patient demographic and fundo/GT status. $H L H S$, Hypoplastic left heart syndrome; $D I L V$, double-inlet left ventricle; hypo RV/TA/PA, hypoplastic right ventricle with tricuspid or pulmonary atresia; $C A V C$, complete atrioventricular canal defect; fundo/GT, fundoplication or gastrostomy tube; ref, reference for HR comparisons.

were performed during the initial hospitalization for the first-stage palliation, with the remainder performed as outpatient, interstage procedures (11 between stage 1 and 2, and 6 between stages 2 and 3). Analysis of these 3 groups (fundoplication or GT performed at stage 1, between stages 1 and 2 , and between stages 2 and 3 ) demonstrated no difference in transplant-free survival $(P=.8)$. There were no operative or perioperative mortalities associated with the gastric procedures.

TABLE 3. Adjusted survival estimates with fundoplication/ gastrostomy tube modeled as a time-dependent covariate

\begin{tabular}{llc}
\hline \multicolumn{1}{c}{ Model } & $\begin{array}{c}\text { HR for fundo/ } \\
\text { GT effect }\end{array}$ & $\boldsymbol{P}$ value \\
\hline $\begin{array}{l}\text { Crude model (just fundo/GT effect) } \\
\begin{array}{c}\text { Model with adjustment for HLHS, } \\
\text { ventricle morphology, birth weight }\end{array}\end{array}$ & $4.29(1.65-11.14)$ & .003 \\
Full adjustment for all covariates & $4.24(1.28-10.78$ & .016 \\
\hline
\end{tabular}

Three patient models. The first model represents the risk of transplant or mortality as reflected by only the effect of fundo/GT regardless of other patient demographics. The second model adjusts the HR for the stated patient demographics. The third model adjusts the HR for all patient demographics. $H R$, Hazard ratio; $G T$, gastrostomy tube; $H L H S$, hypoplastic left heart syndrom.

\section{Transplant-Free Survival Analysis}

Table 2 shows the results of the bivariate survival analysis. The only variables found to be significantly associated with midterm transplant-free survival were the need for fundoplication or GT $(P=.003$; HR, 4.29) and birth weight $(P=.05)$.

Table 3 shows the multivariable survival analysis. The HR for fundoplication or GT alone was $4.29(P=.003)$. After adjusting for the potential confounding effects of all significant covariates, including HLHS diagnosis, ventricular morphology, and birth weight, the HR was similar (HR, $3.72 ; P=.016)$. The third model of the multivariable analysis adjusts the HR for all covariates (HR, 4.24; $P=.014$ ). As shown in Table 3, the HR for fundoplication or GT effect remains significant in all models. By Kaplan-Meier survival analysis, the need for early fundoplication or GT is significantly associated with worse transplant-free survival (Figure 1, $P=.004$ ).

\section{DISCUSSION}

Children with single-ventricle heart disease who undergo staged palliation experience a 2-phase hazard of death or 


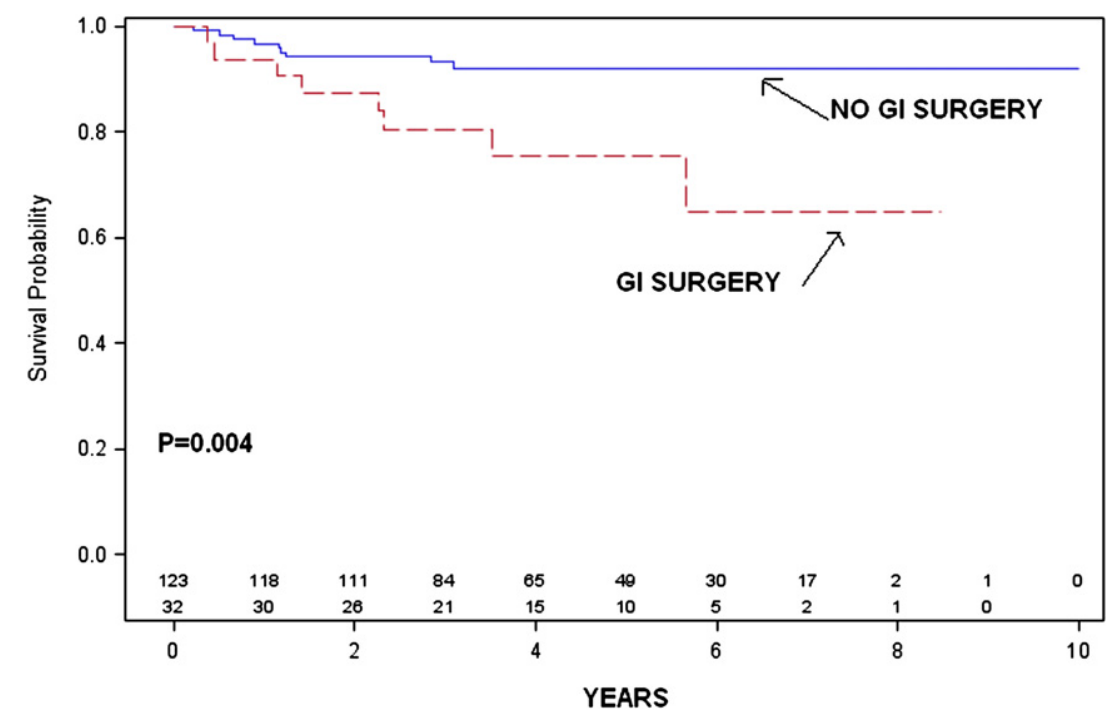

FIGURE 1. Transplant-free survival. Survival of fundoplication/GT and non-fundoplication/GT cases over time. The y-axis is the survival probability, and the $\mathrm{x}$-axis demonstrates years of survival. Patients at risk for the 2 groups are shown along the $\mathrm{x}$-axis. GI surgery indicates performance of a fundoplication or GT within the first 2 years of life.

transplant with a peak risk associated with the first-stage palliation, followed by long-term gradual attrition for survivors of the first stage. Many published studies have investigated the risk factors associated with early mortality after the Norwood procedure. These reports have identified a wide variety of risk factors, including low birth weight, cardiac anatomic subtypes (unbalanced atrioventricular canal or aortic atresia in HLHS), intraoperative variables, and many others. ${ }^{4,7,8}$

The risk factors associated with the late hazard phase for staged palliation have not been well defined. In 1997, Gentles and colleagues ${ }^{3}$ reported the early and late outcomes for the first 500 patients who underwent a Fontan procedure at Children's Hospital Boston between 1973 and 1991. They identified only 2 risk factors associated with late outcome: The presence of a pacemaker before the Fontan was a poor prognostic indicator, whereas certain anatomic subgroups were predictive of better outcomes (morphologic left ventricle with normally related great vessels or single right ventricle excluding heterotaxy and HLHS).

In a more recent study published in 2006, Mitchell and colleagues $^{2}$ at the Children's Hospital of Philadelphia reported their midterm results (9-year median follow-up) for 332 survivors of the Fontan procedure performed between 1992 and 1999. The 10-year transplant-free survival for this selected subgroup who had already survived through the Fontan procedure was $93 \%$. The only significant risk factors for late attrition were the duration of the aortic crossclamp during the Fontan procedure and the duration of chest tube drainage in the postoperative period.

In comparison with these earlier reports, the current study excluded children who died or received transplants before the second-stage palliation but included the second stage and beyond. Although this is a somewhat arbitrary cutoff, it correlates well with the reported demarcation between the early and late hazard phases.

We found that cardiac anatomy and ventricular morphology were associated with the need for fundoplication or GT, with an increased incidence of fundoplication or GT in the HLHS group $(P=.002)$, and a morphologic right ventricle $(P=.008)$. However, neither one of these variables affected midterm transplant-free survival $(P=.4$ and .6 , respectively), whereas the need for fundoplication or GT was significantly associated with poor outcome with an HR of 4.3 $(P=.003)$, and there was little change in this hazard after correction for all covariates.

The question then becomes, what does this mean? Two logical explanations leap immediately to mind. First, the need for a fundoplication or GT early in life may serve as a marker for other unmeasured variables that can affect late survival as a palliated patient with single-ventricle disease. Patient populations who might be enriched in the fundoplication group are those with significant neurologic impairment or an unidentified genetic syndrome. Either one of these characteristics may be associated with an increased risk of late failure. A second possibility is that the presence of GERD early in life that is severe enough to eventually require a fundoplication may result in pulmonary soilage and an increased incidence of chronic pulmonary disease, eventually predisposing this population to elevated pulmonary vascular resistance and Fontan failure. Unfortunately, this study cannot answer this critical question, but it does point to the need for increased vigilance in this particular subgroup. 


\section{Limitations}

This study has several limitations. Although we have been following this cohort prospectively, they do not represent a complete consecutive patient cohort. As a result, the study suffers from the limitations of all retrospective studies and unidentified selection biases. Also, the lack of a comprehensive genetic evaluation of all patients is a clear limitation of the study. Finally, these patients are typical of the complex congenital cardiac population in that they were heterogeneous with regard to the indications for gastric surgery and the timing of these procedures. Because there were many uncontrolled clinical factors, this study can only demonstrate an association between the need for gastric surgery early in life and midterm outcomes. We can make no statements regarding causality.

\section{CONCLUSIONS}

Patients with single-ventricle disease who require early fundoplication or a GT are at increased risk of midterm failure of staged palliation. The implications of this finding are not entirely clear, but this patient population may benefit from increased surveillance.

\section{References}

1. Gordon B, Rodriguez S, Lee M, Chang R. Decreasing number of deaths of infants with hypoplastic left heart syndrome. J Pediatr. 2008;153:354-8.

2. Mitchell M, Ittenbach R, Gaynor J, et al. Intermediate outcomes after the Fontan procedure in the current era. J Thorac Cardiovasc Surg. 2006;131:172-80.

3. Gentles T, Mayer J, Gauvreau K, et al. Fontan operation in five hundred consecutive patients: Factors influencing early and late outcome. J Thorac Cardiovasc Surg. 1997;114:376-91.

4. Stasik C, Goldberg C, Bove E, et al. Current outcomes and risk factors for the Norwood procedure. J Thorac Cardiovasc Surg. 2006;131:412-7.

5. Torres A, DiLiberti J, Pearl R, et al. Noncardiac surgery in children with hypoplastic left heart syndrome. J Pediatr Surg. 2002;37:1399-403.

6. Slater B, Rangel S, Ramamoorthy C, et al. Outcomes after laparascopic surgery in neonates with hypoplastic left heart syndrome. J Pediatr Surg. 2007;42:1118-21.

7. Forbess J, Cook N, Roth S, et al. Ten-year institutional experience with palliative surgery for hypoplastic left heart syndrome. Risk factors related to stage 1 mortality. Circulation. 1995;92(Suppl II):II262-6.

8. Mahle W, Spray T, Wernovsky G, et al. Survival after reconstructive surgery for hypoplastic left heart syndrome: a 15-year experience from a single institution. Circulation. 2000;102(Suppl III):III136-41. 